\title{
Statistical evidence of strain induced breaking of metallic point contacts
}

\author{
M. Alwan ${ }^{1}$, N. Candoni ${ }^{1}$, Ph. Dumas ${ }^{1}$, and H.R. Klein ${ }^{1}$ a \\ Aix Marseille Universite, CNRS, CINAM UMR 7325, 13288 Marseille cedex 9, France
}

Received: date / Revised version: date

\begin{abstract}
A Scanning Tunneling Microscopy in Break Junction regime and a Mechanically Controllable Break Junction are used to acquire thousands of conductance-elongation curves by stretching until breaking and re-connecting $\mathrm{Au}$ junctions. From a robust statistical analysis performed on large sets of experiments, parameters such as lifetime, elongation and occurrence probabilities are extracted. The analysis of results obtained for different stretching speeds of the electrodes indicates that the breaking mechanism of di- and mono-atomic junction is identical, and that the junctions undergo atomic rearrangement during their stretching and at the moment of breaking.
\end{abstract}

PACS. 73.63.Rt electronic transport in nanocontacts - 81.07.Lk fabrication of nanocontacts

\section{Introduction}

During the last decades, electronic properties of atomic sized metallic contacts have interested several groups employing different techniques[1]. First studies were carried out using Scanning Tunneling Microscopy in Break junction regime (STMBJ) [2]. The second most used technique is the Mechanically Controllable Break Junction (MCBJ) developed by Muller et al.[3] in 1992 and based on an earlier design by Moreland and Ekin.[4] Both STM-BJ and MCBJ techniques are based on the stretching of an $\mathrm{Au}$ wire. During stretching, the Au wire gets thinner, and finally breaks leading to two electrodes. Before breaking, at a given bias voltage, the current flowing through the junction is measured as a function of the relative elongation of Au wire. As it is expected in diffusive regime, the current first decreases linearly while the elongation of the wire. However, in the ultimate stage before breaking, while the diameter of the Au wire consists only in a few atoms, the electronic transport becomes ballistic with the conductance given by:

$$
G=\frac{2 e^{2}}{h} \sum_{n=1}^{N} T_{n}
$$

Where $N$ is the number of available channels for the electrons in the junction, $e$ the electron charge, $h$ the Planck's constant and $T_{n}$ the transmission probability of the channels. In junctions of atomic dimensions, conduction channels are directly related to wave functions of the metal [5]. In the particular case of gold, the conductance of a single atom-junction is due to one conductance channel with transmission probability close to unity [6]. Thus, its conductance is close to $2 e^{2} / h$, the conductance quantum $G_{0}$.

\footnotetext{
${ }^{a}$ Author to whom any correspondence should be addressed. klein@ cinam.univ-mrs.fr
}

Therefore, in MCBJ or STM-BJ experiments, a gold monoatomic junction is characterized by a constant conductance of $1 G_{0}$ during the stetching of the junction, i.e. a ballistic plateau at $1 G_{0}$. It is noteworthy that the length of this plateau gives a direct access to the lifetime of the mono-atomic junction. Similarly, the ballistic plateau observed at $2 G_{0}$ is distinctive of a junction containing two atoms in its section.

Such junctions are often employed to bind molecules displaying terminations with a high affinity to metals such as thiols or amino groups [7]. In this purpose, the mono-atomic junction is stretched until it breaks, leading to two Au nano electrodes. Then in the presence of molecules, these ones can graft on the electrodes and the conductance of the Au-molecule-Au junction are measured, giving access to single molecule conductance [8]. However such studies require stabilizing junctions for a period as long as possible. Thus, the understanding of the Au junction stability and breaking mechanism is crucial.

Tsutsui et al have studied the dependence of the lifetime of monoatomic gold contacts on the stretching speed of the electrodes [9]. They showed that for stretching speed higher than $1 \mathrm{~nm} . \mathrm{s}^{-1}$, lifetime is limited by the strain applied to the junction. Similar results were obtained for molecular junction by Huang et al [10]. For lower stretching speed, lifetime is rather limited by thermal motion, leading to discrete conductance fluctuations depending on the temperature of the experiment [11].

Literature generally focuses on the ultimate ballistic plateau at $1 G_{0}$, whereas the properties of the junction may depend on its earlier configuration. Therefore, in this work we explore as much ballistic plateaus at $1 G_{0}$ as at $2 G_{0}$. Our study concerns the behavior of mono- and di-atomic junctions associated to 1 and $2 G_{0}$ conductances, respectively, at different stretching speeds in ambient conditions. Our specificity is the comparison of results obtained with two complementary techniques STM- 


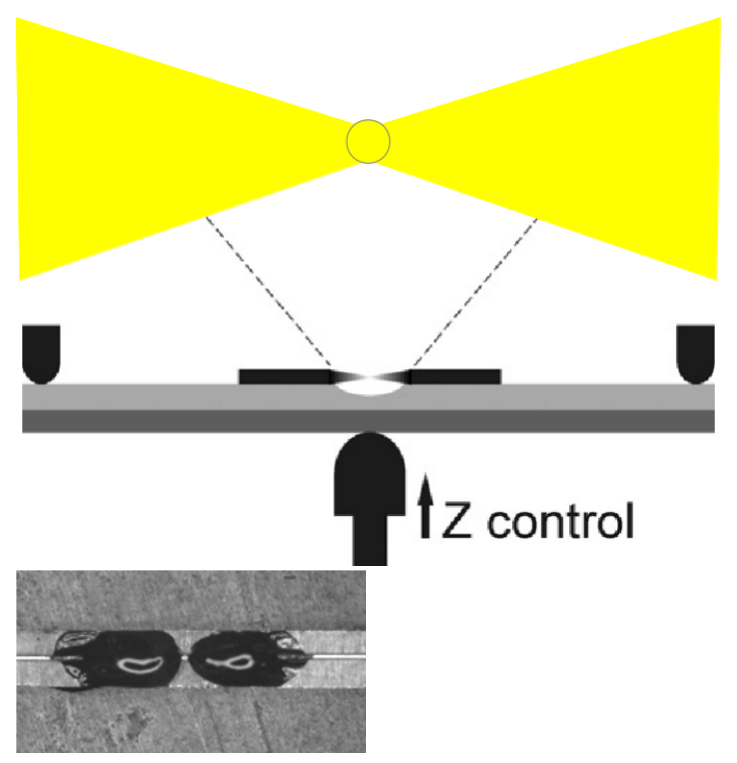

Fig. 1. Schematics of our home made MCBJ. Top : The basic principle consists in stretching the wire by bending the substrate with a mechanical actuator, until the junction breaks and gives two separate electrodes. Relaxing the force on the elastic substrate by back-bending allows the junction to be closed again. The mechanical actuator is composed of a stepping motor and a piezoelectric transducer (PZT) in series. Down : A thin Au wire (diameter $125 \mu \mathrm{m}$ ) is glued onto a phosphorous bronze bending beam with two droplets of epoxy.

BJ and MCBJ, developed in the laboratory, in order to avoid artifacts in these delicate measurements.

A great deal of effort is made in this paper to investigate experimental results through an objective statistical approach in order to highlight geometry and mechanical properties of $\mathrm{Au}$ junction. This is made possible by the ability to repeat the atomic contact experiments thousands of times using break junction setups (MCBJ or STM-BJ).

\section{Experiments}

\subsection{Set-up}

In STM-BJ, Au junctions are obtained by indenting an Au surface with a gold tip. Surfaces are prepared by electron beam evaporation under ultra high vacuum conditions of a $100 \mathrm{~nm}$ layer of $\mathrm{Au}$ with a preferential [111] orientation [12], while gold tips are electrochemically etched from $0.25 \mathrm{~mm}$ gold wire (99.99\%, Goodfellow).

In our home-made MCBJ (sketched in figure 1), a thin Au wire (diameter $250 \mu \mathrm{m}$ ) is glued onto a phosphorous bronze bending beam with two droplets of epoxy (Stycast 2850FT, curing agent $24 \mathrm{LV}$ ). The basic principle consists in stretching the wire by bending the substrate with a mechanical actuator, until the junction breaks and gives two separate electrodes. Relaxing the force on the elastic substrate by back-bending allows the junction to be closed again. The mechanical actuator is composed of a stepping motor and a piezoelectric transducer (PZT) in series, which permits to control the process by ramping the $\mathrm{z}$ piezovoltage. Because the wire is fixed, the vertical movement of the PZT is demagnified, allowing accurate control of wire stretching (elongation). The relative elongation can reach a resolution in the range of 5 picometers. Moreover, the MCBJ exhibits an outstanding mechanical stability $(<5 \mathrm{pm} / \mathrm{s}$ drift of the electrodes after breaking at room temperature for our setup). This stability results from the reduction of the mechanical loop which connects one electrode to the other, from centimeters, in the case of an STM scanner, to approximatively $0.2 \mathrm{~mm}$ in our MCBJ. The drawback is that the demagnification factor depends on the geometrical parameters of the sample, mainly on the distance between the glue droplets. It is thus difficult to obtain an absolute calibration of the relative displacement of the electrodes. An accurate calibration method using the Gundlach oscillations in the tunnel current has been proposed by Kolesnychenko et al [13]. Unfortunately this method is not suitable for operation in ambient conditions. To circumvent this potential calibration issue, we have combined the MCBJ measurements with STM-BJ ones, where displacements are easily calibrated.

For the two setups, experiments are carried out in air using a bias voltage of the electrodes of $130 \mathrm{mV}$. The current is measured using a DLPCA-200 I/V converter (Femmto Messtechnik). Conductance versus elongation traces are recorded using a dedicated software described in detail elsewhere [14]. Briefly, while the MCBJ or STM-BJ regulation loop is maintained off, the two electrodes are brought into contact prior to breaking off the junction by pulling the electrodes away with variable stretching speeds. Conductance data are simultaneously acquired at a sampling frequency of $10 \mathrm{kHz}$. The regulation loop is then activated for approximatively $1 \mathrm{~s}$ and the cycle is repeated automatically thousands of times.

\subsection{Methods}

The recorded curves show ballistic plateaus in the transition between contact and vacuum tunneling. The quantization of conductance appears at approximately integer multiples of the Landauer conductance quantum, $G_{0}=2 e^{2} / h$ as expected. However, some plateaus in the conductance curves cannot be unambiguously identified to integer multiples of the conductance quantum, $n G_{0}$. In an attempt for an objective analysis of the data, we identify conductance plateaus by the abrupt changes of conductance, which separate them. The plateaus shorter than three consecutive acquisition points $(0.2 \mathrm{~ms})$ are discarded. From the acquisition of thousands of individual conductance curves of the same junction, histograms of conductance values are constructed, as shown on figure 2,

More advanced statistics are performed after acquisition. The algorithms developed for these tasks are described elsewhere [14]. They allow the extraction of occurences, average conductances and lifetimes of conductance plateaus. The same algorithms are used to analyse STM-BJ and MCBJ experiments.

\section{Results and discussion}

We have tested, using STM-BJ and MCBJ, stretching speeds of the electrodes in the range from 0.5 to $1000 \mathrm{~nm} . \mathrm{s}^{-1}$. In the recorded conductance-elongation curve, we focus on plateaus 


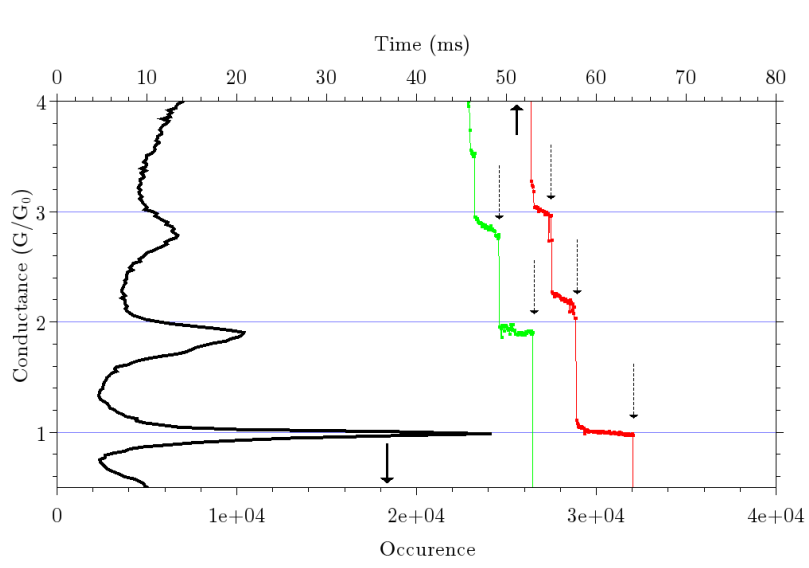

Fig. 2. Typical histogram of conductance constructed from a set of 9000 conductance vs elongation traces recorded using the STMBJ at a stretching speed of $142 \mathrm{~nm} \cdot \mathrm{s}^{-1}$ (bias $130 \mathrm{mV}$ ). Two conductance vs elongation traces are also shown. On these traces conductance plateaus are separated by abrupt changes of conductance marked by the dashed arrows.

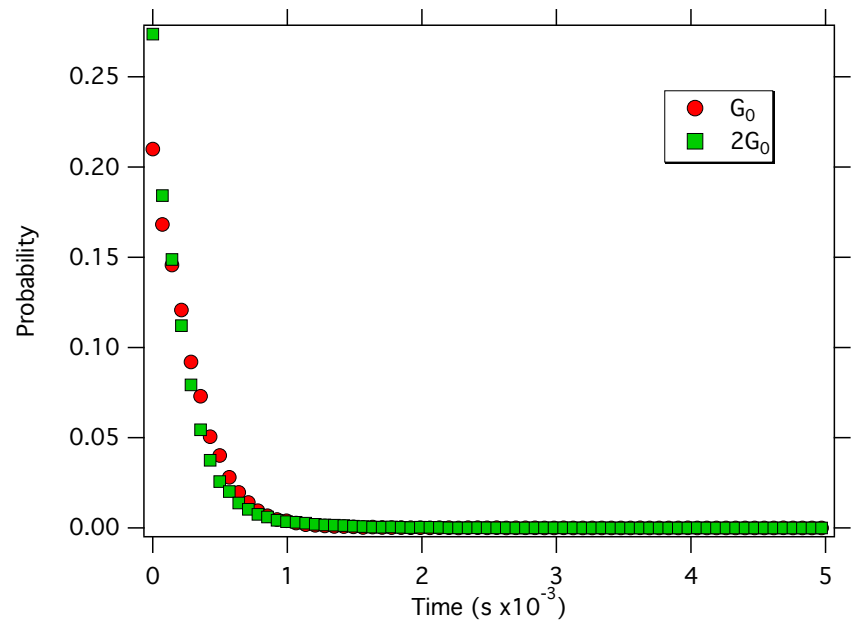

Fig. 3. Histogram of lifetimes of $G_{0}$ and $2 G_{0}$ plateaus recorded using the MCBJ at a stretching speed of $142 \mathrm{~nm} . \mathrm{s}^{-1}$ (bias $130 \mathrm{mV}$ ). Both histograms are obtained from sets of 75000 elongation curves, and can be described as an exponential decay law with similar time constants. STM-BJ experiments exhibit a similar behaviour.

at $1 G_{0}\left(0.8 G_{0}<G<1.2 G_{0}\right)$ and $2 G_{0}\left(1.6 G_{0}<G<\right.$ $2.4 G_{0}$ ), which are attributed to mono- and di-atomic sections of the junction, respectively.

\subsection{Lifetimes of mono- and di-atomic junctions}

As conductance measurement is acquired at a constant stretching speed, the elongation of the contacts leading to ballistic plateaus at $1 G_{0}$ and $2 G_{0}$ is directly proportionnal to junctions' lifetime, reflecting their stability. From sets of thousands conductance curves, we can plot histograms of lifetimes of monoand di-atomic junction lifetimes.

As shown in figure 3, the occurrence of plateaus lifetime typically leads to an exponential decay law given by the general

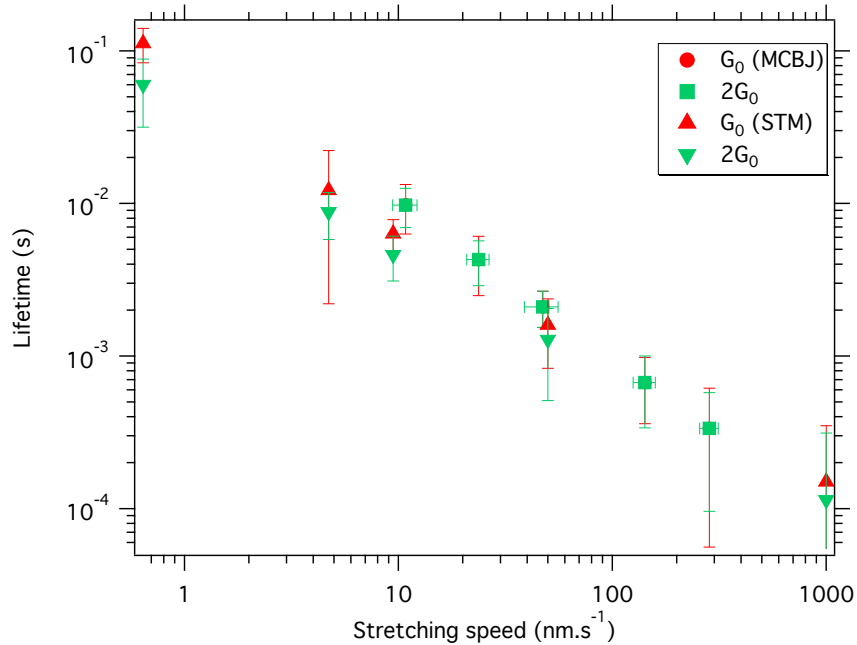

Fig. 4. Lifetimes of ballistic plateaus at 1 and $2 G_{0}$ vs stretching speed of the electrodes obtained with MCBJ (circles and squares) and STM$\mathrm{BJ}$ (up and down triangles) at a bias of $130 \mathrm{mV}$. Values calculated from 75000 experiments. Horizontal error bars on MCBJ points represents the uncertainty in the calibration of the relative displacement of the electrodes.

expression

$$
p(t)=\frac{\exp (-t / \tau)}{\tau}
$$

where $\tau$ corresponds to the mean value of lifetime. This exponential decrease is a clear indication of the random nature of the breaking process, as we have already reported in [14]. Without any further investigations, this random process suggests that mono- and di-atomic junctions' breaking are driven either by thermal motion, by a random external perturbations or by the constraint applied to the junction, which would provide the system enough energy to break.

The dependence of the lifetime of ballistic plateaus at 1 and $2 G_{0}\left(\tau_{1}\right.$ and $\tau_{2}$ respectively) on the stretching speed shown in figure 4,indicate that the breaking is due to the strain applied to the junction. Indeed the strain increase with the stretching speed, and thus the lifetime decreases. Noteworthy, $\tau_{1}$ and $\tau_{2}$ exhibits similar values, and the same dependence on the stretching speed.

This result is in excellent quantitative agreement with data for ballistic plateaus at $1 G_{0}$ previously published by Tsustui [9] and Huang [10], using an MCBJ and a STM-BJ respectively.

We can imagine that the junction breaks like a constrained rope, one strands (conduction channel) after the other at a given lengthening [15]. In such a scenario, a diatomic junction displays two parallel conduction channels for the electrons flowing trough the junction. One of these channels breaks under strain, leading to a mono-atomic junction. We would then expect $\tau_{1}$ to be much shorter than $\tau_{2}$ because the last channel has already been stretched in the di-atomic junction before it breaks. However, $\tau_{1}$ and $\tau_{2}$ show quite close values and the same dependence on $v$, suggesting that the breaking mechanism is not sequential, and has the same origin for mono- and di-atomic junctions. 


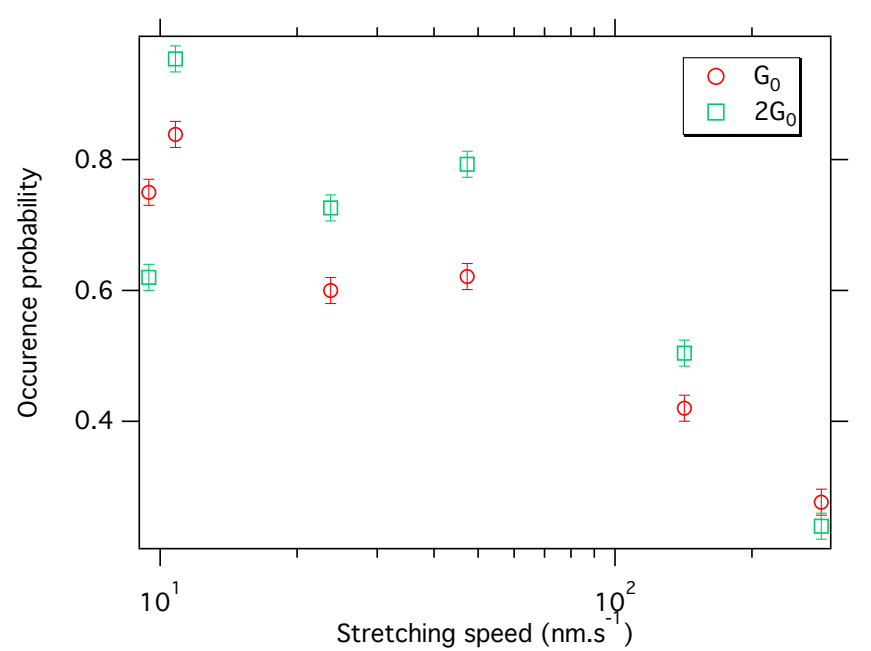

Fig. 5. Plateaus occurence probabilities at 1 and $2 G_{0}$ vs stretching speed. These values are obtained from sets of 75000 conductance curves for each stretching speed using MCBJ and STM-BJ at a bias of $130 \mathrm{mV}$. Increasing the stretching speed (and thus the strain applied to the junction), lead to a decrease of the occurence probabilities of conductance plateaus.

\subsection{Occurence of mono- and di-atomic junctions}

Our advanced statistical analysis allows extracting not only lifetimes but also occurence probabilities of ballistic plateaus at 1 and $2 G_{0}, P_{1}$ and $P_{2}$ respectively. The dependence of these probabilities on the stretching speed is shown on figure 5 .

This curve shows that the ocurences of ballistic plateaus dramatically decrease while the stretching speed (and thus the constraint) increases.

In addition, on figure 6 , the dependence of the product of the probabilities $P_{1} . P_{2}$ on $P_{1 \& 2}$, the joint probability to observe conductance plateaus at 1 and $2 G_{0}$ on the same conductance curve, is plotted. These probabilities show very close values for all the stretching speed explored in this study with the STM-BJ and MCBJ setups. It points out that the occurences of ballistic plateaus are statistically independent events. In the case of sequential breaking of di- and mono-atomic junctions, plateaus at 1 and $2 G_{0}$ must appear together in conductancetime curves leading to correlated occuring probabilities. The observed statistical independence means that the plateau at 1 $G_{0}$ exists wether the plateau at $2 G_{0}$ exists or not, and viceversa, as shown by the combination of the probabilities $P_{1}$ and $P_{1 \& 2}$. Using these values we can calculate that $50 \%$ of the 1 $G_{0}$ events are subsequent to $2 G_{0}$ events at the stretching speed of $142 \mathrm{~nm} . \mathrm{s}^{-1}$, to be compared to $23 \%$ at $284 \mathrm{~nm} . \mathrm{s}^{-1}$ and 60 $\%$ at $23.7 \mathrm{~nm} . \mathrm{s}^{-1}$ for instance.

Consequently, the di-atomic junction is certainly not composed of two conduction channels in parallel, which would break sequentially. But rather a more complicated atomic structure implying a width of two atoms in its thinner part, as confirmed by tight-binding molecular dynamic $[16,17,14]$. These simulations show that the junction displays an asymetric structure during stretching, meaning that the strain applied to the atomic bonds is not homogeneous.

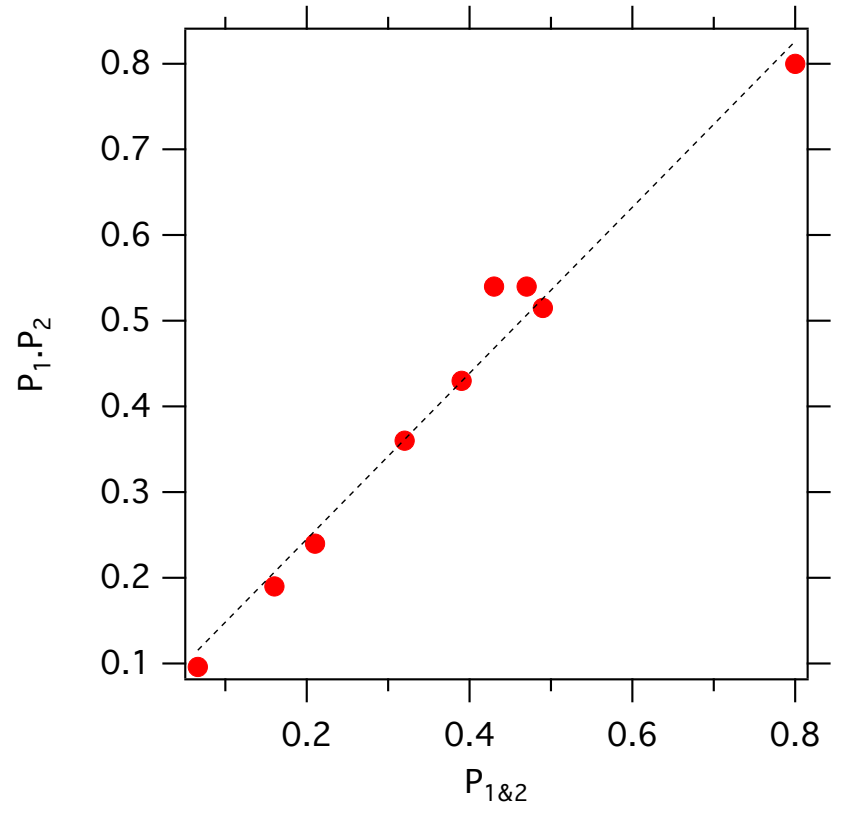

Fig. 6. Product of the probabilities to observe a plateau at $G_{0}\left(P_{1}\right)$ times the probability to observe a plateau at $2 G_{0}\left(P_{2}\right)$ vs the joint probability $P_{1 \& 2}$ to observe both plateaus during the same experiment. This graph gathers the results obtained from the STM-BJ and MCBJ experiments for the whole stretching speed range of the present study. Dashed line : $P_{1} \cdot P_{2}=P_{1 \& 2}$

\subsection{Breaking mechanism}

During stretchnig, the junction stiffness increases with the accumulated elastic energy. Then, the only way to relax a part of this excess of energy is a plastic deformation, by the rupture of the most strained bond. This occurs for given elongations, independent of the stretching speed, $0.1-0.15 \mathrm{~nm}$ in our study. Several experimental studies at low temperature [18] and in ambient conditions [10] reported an elongation at rupture of 0.1 to $0.25 \mathrm{~nm}$ corresponding to inter-atomic distance between gold atoms , in good agreement with molecular dynamics simulations. Thus, the junction has to be considered more as a contact than as a chain of atoms. G. Rubio-Bollinger estimated a rupture force of $1.5 \pm 0.5 \mathrm{nN}$ [19]. By considering the junction as a spring, the ration between this force and elongation at rupture leads to a spring constant of $10-15 \mathrm{~N} \cdot \mathrm{m}^{-1}$, and an energy release at rupture of $0.2-0.7 \mathrm{eV}$. Numerical simulations of junction breaking are in excellent agreement with experimental values [20].

Our study points out that similar results are obtained both for mono- and di-atomic junctions, indicating that their breaking mechanism is identical. As the junction undergoes plastic deformation, the corresponding energy release can lead either to atomic reconfiguration of the junction or to breaking of the remaining bonds. Hence the conductance elongation curve, after a $2 G_{0}$ conductance plateau, will display either a rupture or a conductance plateau at $1 G_{0}$ corresponding to a junction with a structure not correlated with the previous one. This fully explains the fact that 1 and $2 G_{0}$ conductance plateaus are statistical independent events. 


\section{Conclusion}

A MCBJ and a STM-BJ have been used to acquire thousands of conductance - elongation curves by stretching until breaking and re-connecting Au junctions. Data on ballistic plateaus at $1 G_{0}$ and $2 G_{0}$ are extracted and statistically analysed, in order to explain the breaking of mono- and di-atomic Au junctions in ambient conditions.

Our study relies on a robust statistical analysis performed on large sets of experiments. Moreover, it combines results obtained with two complementary techniques : STM-BJ and MCBJ. The excellent agreement between the results, strengthen the analysis and ensures the lack of artifacts in lifetime measurements related either to external perturbations (vibrations, thermal drift) in STM-BJ experiments, or calibration issues in MCBJ ones.

The dependence of the lifetime of gold junctions on the stretching speed of the electrodes showed that for stretching speed higher than $1 \mathrm{~nm} . \mathrm{s}^{-1}$, lifetime is limited by the strain applied to the junction. This is in agreement with previously published works focusing on mono-atomic junctions. The present study extends this result to di-atomic junctions indicating that their breaking mechanism is identical. Moreover, the analysis of occurrence probabilities of the ballistic plateaus gives an experimental evidence that junctions undergo atomic rearrangement during their stretching and at the moment of breaking.

To our knowledge, it is the first time that a statistical approach provides such complementary informations on breaking mechanism of Au nano-junctions.

\section{References}

1. Nicolas Agraï, Alfredo Levy Yeyati, and Jan M. van Ruitenbeek. Quantum properties of atomic-sized conductors. Physics Reports, 377(2-3):81-279, April 2003.

2. J. K. Gimzewski and R. Moller. Transition from the tunneling regime to point contact studied using scanning tunneling microscopy. Phys. Rev. B, 36(2):1284-1287, July 1987.

3. C. J. Muller, J. M. van Ruitenbeek, and L. J. de Longh. Conductance and supercurrent discontinuities in atomic-scale metallic constrictions of variable width. Phys. Rev. Lett., 69(1):140143, 1992.

4. John Moreland and J. W. Ekin. Electron tunneling experiments using nb-sn "break" junctions. Journal of Applied Physics, 58(10):3888-3895, 1985.

5. E. Scheer, N. Agrait, J. C. Cuevas, A. Levy Yeyati, B. Ludolph, A. Martin-Rodero, G. B. Bollinger, J. M. van Ruitenbbek, and C. Urbina. The signature of chemical valence in the electrical conduction through a single atom contact. Nature, 394:154-157, 1998.

6. L. de la Vega, A. Martin-Rodero, A. Levy Yeyati, and A. Saul. Different wavelength oscillations in the conductance of $5 \mathrm{~d}$ metal atomic chains. Phys. Rev. B, 70(11):113107-, September 2004.

7. Fang Chen, Joshua Hihath, Zhifeng Huang, Xiulan Li, and N. J. Tao. Measurement of single-molecule conductance. Annual Review of Physical Chemistry, 58(1):535-564, May 2007.

8. T. Leoni, Alwan M., H. Klein, N. Candoni, A. Lereu, and P. Dumas. Tools for conductance measurements of metallic and molecular nanowires. Glob. J. Phys. Chem., 2:97-103, 2011.
9. M. Tsutsui, K. Shoji, M. Taniguchi, and T. Kawai. Formation and self-breaking mechanism of stable atom-sized junctions. Nano Letters, 8(1):345-349, 2008.

10. M. Huang, F. Chen, P.A. Bennett, and N.J. Tao. Single molecule junctions formed via au thiol contact: Stability and breakdown mechanism. Journal of the American Chemical Society, 129(43):13225-13231, October 2007.

11. H. Klein, T. Leoni, R. Zoubkoff, P. Dumas, and A. Saul. Conductance fluctuations in gold point contacts: an atomistic picture. Nanotechnology, 23(23):235707, 2012.

12. H. Klein, W. Blanc, R. Pierrisnard, C. Fauquet, and P. Dumas. Self-assembled monolayers of decanethiol on au(111)/mica. European Physical Journal B, 14(2):371-376, 2000.

13. O. Yu. Kolesnychenko, O. I. Shklyarevskii, and H. van Kempen. Calibration of the distance between electrodes of mechanically controlled break junctions using field emission resonance. Review of Scientific Instruments, 70(2):1442-1446, 1999.

14. Thomas Leoni, Remi Zoubkoff, Sabrina Homri, Nadine Candoni, Petar Vidakovic, Alain Ranguis, Hubert Klein, Andres Saul, and Philippe Dumas. Are conductance plateaus independent events in atomic point contact measurements? a statistical approach. Nanotechnology, 19(35):355401-, 2008.

15. G. Rubio-Bollinger, S. R. Bahn, N. Agraït, K. W. Jacobsen, and $\mathrm{S}$. Vieira. Mechanical properties and formation mechanisms of a wire of single gold atoms. Phys. Rev. Lett., 87(2):026101, June 2001.

16. P. Z. Coura, S. B. Legoas, A. S. Moreira, F. Sato, V. Rodrigues, S. O. Dantas, D. Ugarte, and D. S. Galvao. On the structural and stability features of linear atomic suspended chains formed from gold nanowires stretching. Nano Letters, 4(7):1187-1191, 2004.

17. M. Dreher, F. Pauly, J. Heurich, J. C. Cuevas, E. Scheer, and P. Nielaba. Structure and conductance histogram of atomic-sized au contacts. Phys. Rev. B, 72(7):075435, August 2005.

18. A. I. Yanson, G. Rubio Bollinger, H. E. van den Brom, N. Agrait, and J. M. van Ruitenbeek. Formation and manipulation of a metallic wire of single gold atoms. Nature, 395(6704):783-785, October 1998.

19. G. Rubio, N. Agraït, and S. Vieira. Atomic-sized metallic contacts: Mechanical properties and electronic transport. Phys. Rev. Lett., 76(13):2302-2305, March 1996.

20. P. Vélez, S. A. Dassie, and E. P. M. Leiva. When do nanowires break? A model for the theoretical study of the long-term stability of monoatomic nanowires. Chemical Physics Letters, 460(13):261-265, July 2008. 